\title{
Effects of the low lying Dirac modes on the spectrum of ground state mesons
}

\author{
C.B. Lang \\ Institut für Physik, FB Theoretische Physik, Universität Graz, A-8010 Graz, Austria \\ E-mail: christian.lang@uni-graz.at \\ Mario Schröck* \\ Institut für Physik, FB Theoretische Physik, Universität Graz, A-8010 Graz, Austria \\ E-mail: mario.schroeckeuni-graz.at
}

The lowest eigenmodes of the Dirac operator are related to the dynamical breaking of the chiral symmetry in Quantum Chromodynamics (QCD). In our work we construct quark propagators which exclude a varying number of the lowest Dirac eigenmodes and study the influence thereof on meson correlators and the meson spectrum. That procedure partially restores the chiral symmetry (in the valence sector) and we observe degeneracies in the spectrum while confinement seems not to be affected.

XXIX International Symposium on Lattice Field Theory

July 10-16, 2011

Squaw Valley, Lake Tahoe, California

${ }^{*}$ Speaker. 


\section{Motivation}

The Banks-Casher relation [1] associates the density of the smallest non-zero eigenvalues to the chiral condensate. The importance of the lowest Dirac eigenmodes in QCD has been investigated within several studies: in [2-4] it was shown that the pseudoscalar and axial vector correlators can be approximated in the medium to large temporal distance region when taking only the lowest Dirac eigenmodes as input for the quark propagators into account. In [4-7] this was used to improve the signal of hadron correlators by averaging the low mode contribution to the quark propagator over all possible positions of the sinks on the lattice. The authors of $[4,5]$ studied the influence of low mode removal in the quenched approximation using the overlap operator $[8,9]$.

In our study [10] we construct meson correlators out of reduced quark propagators which exclude a varying number of the lowest Dirac eigenmodes. Using these propagators we compute meson masses and we study the possible recovery of the degeneracies of (would be) chiral partners in the spectrum. Moreover this gives us insight on the role of the lowest Dirac eigenmodes for confinement and thus on the relation of chiral symmetry and confinement. Our modification is applied only to the valence quark sector; the configurations have been obtained with two dynamical light quark flavors without truncation.

The two lightest quark flavors in QCD are much lighter than the typical QCD scale. Neglecting the mass of these two light quarks, the Lagrangian is invariant under the symmetry group

$$
\mathrm{SU}(2)_{L} \times \mathrm{SU}(2)_{R} \times \mathrm{U}(1)_{V} \times \mathrm{U}(1)_{A}
$$

The single flavor symmetry $\mathrm{U}(1)_{V}$ conserves the baryon number density. The axial vector part of the chiral symmetry $\mathrm{SU}(2)_{L} \times \mathrm{SU}(2)_{R}$ mixes states with opposite parity; the nondegenerate masses of parity partners in nature indicate the dynamical breaking of the axial symmetry whereas the isospin symmetry is (approximately) preserved in the vacuum. The single flavor axial symmetry $\mathrm{U}(1)_{A}$ mixes the currents of the same isospin but opposite parity. The latter is not only broken spontaneously but also explicitly by the anomaly.

We will investigate the influence of low mode reduction on the pseudoscalar, vector, scalar and axial vector currents with the interpolating fields given in Table 1 . In a chirally symmetric world, the currents number three and six of the table would get mixed via the isospin axial transformation and the currents one and five would get mixed via the single flavor axial transformation $\mathrm{U}(1)_{A}$.

We did not include isoscalars in our work (these need disconnected terms, notoriously dif-

\begin{tabular}{|c|c|c|c|}
\hline$\#$ & meson & $J^{P C}$ & interpolator \\
\hline \hline 1 & $\pi$ & $0^{-+}$ & $\bar{u} \gamma_{5} d$ \\
\hline 2 & $\pi$ & $0^{-+}$ & $\bar{u} \gamma_{4} \gamma_{5} d$ \\
\hline 3 & $\rho$ & $1^{--}$ & $\bar{u} \gamma_{i} d$ \\
\hline 4 & $\rho$ & $1^{--}$ & $\bar{u} \gamma_{4} \gamma_{i} d$ \\
\hline 5 & $a_{0}$ & $0^{++}$ & $\bar{u} d$ \\
\hline 6 & $a_{1}$ & $1^{++}$ & $\bar{u} \gamma_{i} \gamma_{5} d$ \\
\hline
\end{tabular}

Table 1: The mesons and the corresponding interpolating fields studied in this work. ficult to compute), otherwise we could additionally study the possible recovery of degeneracies in the spectrum between the $h_{1}$ and current four, between $f_{0}$ and current one as well as between the $\eta$ and current five, which all would pairwise mix via the axial vector transformation of the chiral symmetry. For a detailed discussion of the mixing of different currents under the chiral symmetry see [11]. 


\section{Method}

Most lattice Dirac operators $D$ (an exception is the overlap operator $[8,9]$ ) are nonnormal operators, thus one has to distinguish between left eigenvectors $\left\langle L_{i}\right|$ and right eigenvectors $\left|R_{i}\right\rangle$ to a given (complex valued) eigenvalue $\lambda_{i}$. Then the spectral representation of $D$ reads

$$
D=\sum_{i=1}^{N} \lambda_{i}\left|R_{i}\right\rangle\left\langle L_{i}\right|
$$

The hermitian Dirac operator $D_{5} \equiv \gamma_{5} D$ is normal and has real eigenvalues $\mu_{i}$. Alternatively $D$ can be written in terms of the spectral representation of $D_{5}$ in the following way:

$$
D=\sum_{i=1}^{N} \mu_{i} \gamma_{5}\left|v_{i}\right\rangle\left\langle v_{i}\right|
$$

We want to calculate the meson spectrum using reduced quark propagators which leave out a part of the low lying Dirac spectrum. Therefore we split the quark propagator $S=D^{-1}$ into a low mode part (lm) and a reduced part (red), e.g., using the eigenvalues and eigenvectors of $D_{5}$,

$$
S=\sum_{i \leq k} \mu_{i}^{-1}\left|v_{i}\right\rangle\left\langle v_{i}\left|\gamma_{5}+\sum_{i>k} \mu_{i}^{-1}\right| v_{i}\right\rangle\left\langle v_{i}\right| \gamma_{5} \equiv S_{\operatorname{lm} 5(k)}+S_{\operatorname{red} 5(k)} .
$$

Hence we can obtain the reduced part of the propagator by subtracting the low mode part from the full propagator

$$
S_{\text {red5 }(k)}=S-S_{\operatorname{lm} 5(k)} .
$$

Note that the low mode part has to operate on the same sources as $S$ in order to obtain the correct reduced propagators. The corresponding separation using the left and right eigenvectors and the eigenvalues of $D$ can be done in an analogous way.

\section{The setup}

For our calculation we used 161 gauge field configurations [12, 13] of lattice size $16^{3} \times 32$. The lattice spacing is $a=0.144(1) \mathrm{fm}$ and thus the spatial lattice size $L=2.3 \mathrm{fm}$. The configurations are obtained with two light degenerate dynamical quark flavors with an AWI-mass of $m=15.3$ (3) MeV and a corresponding pion mass of $m_{\pi}=322(5) \mathrm{MeV}$. For the dynamical quarks of the configurations as well as for the valence quarks of our study the so-called Chirally Improved Dirac operator $[14,15]$ has been used. This operator is an approximate solution to the Ginsparg-Wilson equation and therefore exhibits better chiral properties than the Wilson Dirac operator while being less expensive by an order of magnitude in comparison to the chirally exact overlap operator.

On these configurations we computed the lowest 256 eigenvalues of $D$ and the lowest 512 eigenvalues of $D_{5}$, see Fig. 1, using ARPACK which is an implementation of the Arnoldi method to calculate a part of the spectrum of arbitrary matrices [16].

In Fig. 2 we show histograms of the lowest 256 eigenvalues of $D$ and $D_{5}$. Additionally in the same figure the integrals over these histograms are given. 

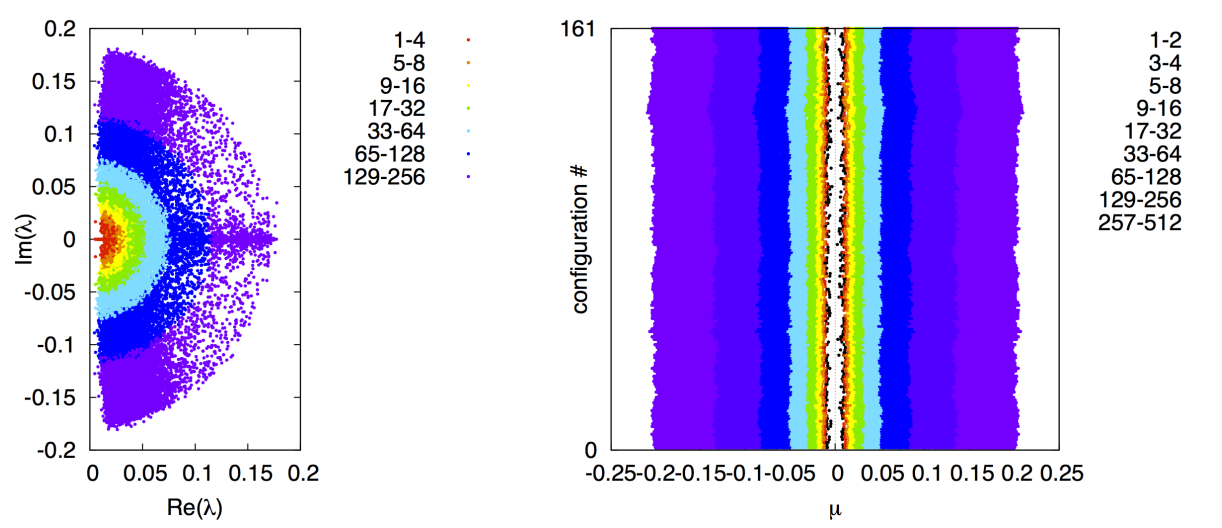

Figure 1: The lowest 256 eigenvalues of $D$ (left) and the lowest 512 eigenvalues of $D_{5}$ (right) for all 161 configurations.
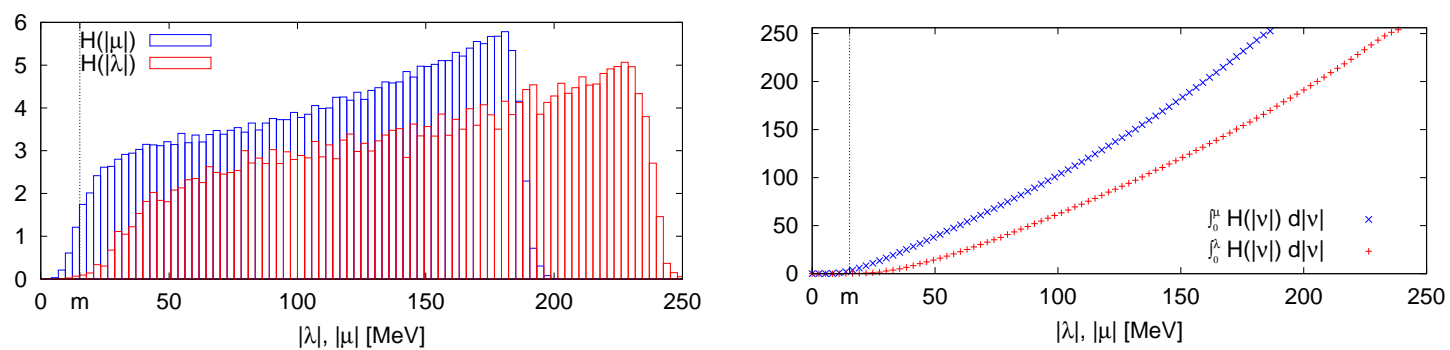

Figure 2: L.h.s.: histograms of the lowest 256 eigenvalues of $D$ (red) and $D_{5}$ (blue). R.h.s.: integrated histograms.

\section{Results}

Before we study the effect of low mode reduction, we look at meson correlators which are build solely out of the low mode part of the propagator, $S_{\operatorname{lm}(k)}$ or $S_{\operatorname{lm} 5(k)}$, respectively (following the ideas in [2-4]). In Fig. 3 we show the pion and rho correlators (interpolators one and three of Table1) from the low mode only propagators $S_{\operatorname{lm}(128)}$ and $S_{\operatorname{lm} 5(32)}$ in comparison with the correlators from full propagators. As can be seen in both cases, four times less eigenvalues of $D_{5}$ in comparison to eigenvalues of $D$ are already sufficient to obtain a similar quality approximation of the full correlators. Since the eigenvalues of $D_{5}$ seem to be more effective for the low mode saturation of meson correlators, we will concentrate on the eigenvalues of $D_{5}$ throughout the rest of this work.

In Fig. 5 we show the correlators under $D_{5}$ low mode reduction in comparison to the correlators from full quark propagators. We find that negative parity mesons (currents 1-4) become heavier under low mode reduction whereas positive parity mesons (currents 5-6) at first become lighter and after subtracting more than 8-16 modes, their mass begins to increase as well. Except for the pion from current one, all correlators still show a clear exponential form (at least as good as for the full propagators) from which we conclude that we are still confronted with bound particles. The loss of the exponential behavior in current one can qualitatively be explained with the strong sensitivity of the pion on the lowest Dirac modes in the sense that very few modes are sufficient to saturate the pseudoscalar correlator in the medium to large time region. Moreover we find that low mode 

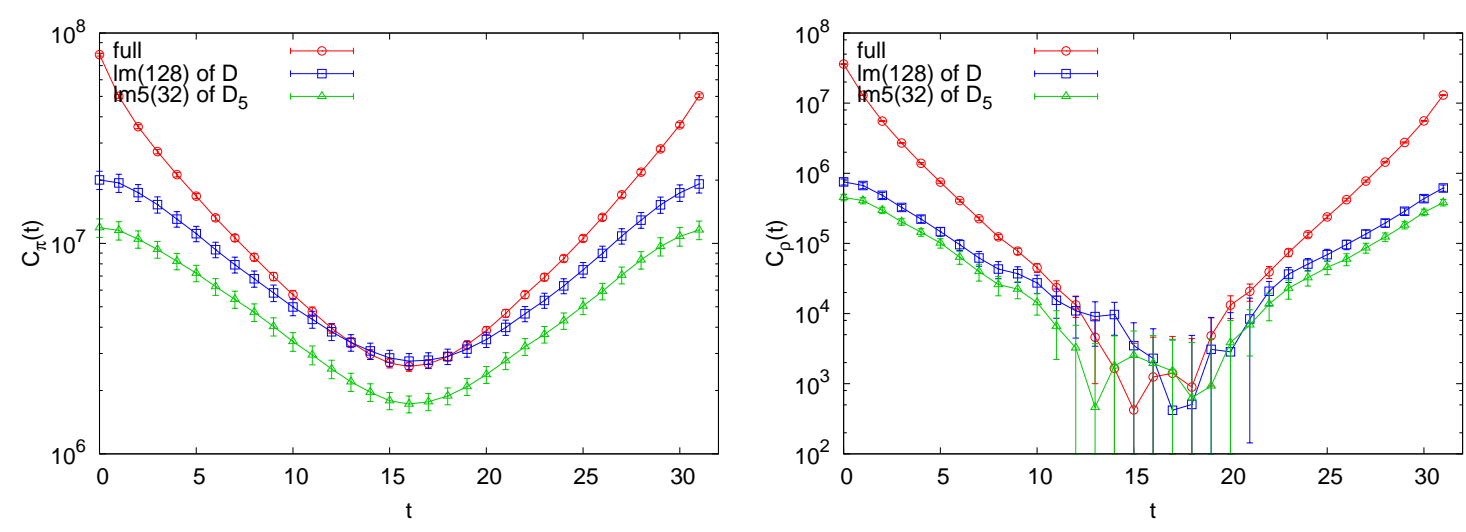

Figure 3: Low mode contribution to the pion (left) and rho (right) correlators.

removal reduces the noise of the correlators which is most obvious for the positive parity currents.

We performed effective mass fits for all the reduced correlators and combined the resulting mass values, as a function of the reduction level, in Fig. 4. For the pion from current one we were only able to perform fits for very low reduction levels. The masses of current three and six are found to be degenerate from truncation level 16 onwards which corresponds to having subtracted all eigenvalues with a magnitude equal to or less than twice the bare quark mass of our setting. Furthermore current four becomes degenerate with currents three and six which hints towards a larger degeneracy which also might include the $h_{1}$ which we did not calculate. Whereas we observe chiral restoration

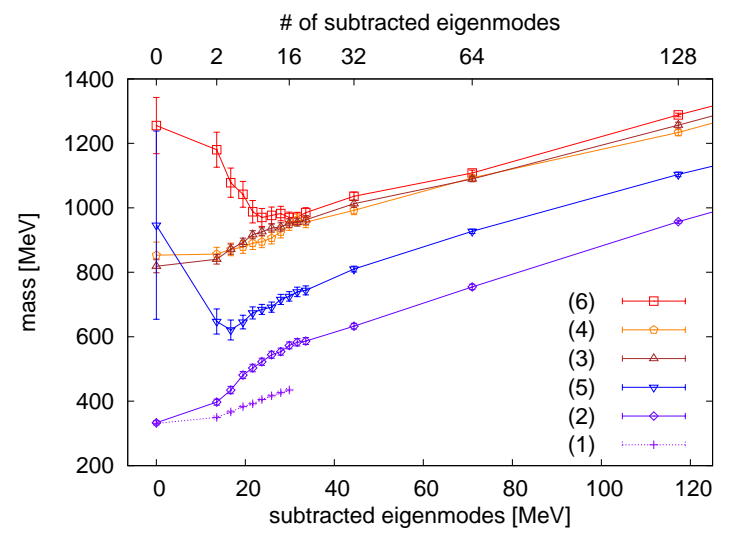

Figure 4: The change of the masses of the mesons under low mode removal of the ingoing quark propagators. The labeling number corresponds to the currents from Table 1.

in the $J=1$ states, we cannot draw any conclusions for $J=0$ states since the would-be chiral partner of the $a_{0}$ (current five) is the pion (current one) which gets destroyed under low mode reduction. Current two which couples to the pion due to PCAC (partially conserved vector current) does not mix with the scalar so there is no reason these two states should become degenerate. For further discussion see [10].

\section{Conclusions}

We studied the isovector meson spectrum using valence quark propagators where a varying number of the lowest Dirac eigenmodes has been removed. The density of the lowest eigenmodes is related to the spontaneous breaking of the chiral symmetry [1]. We find restoration of the chiral symmetry in $J=1$ states when only 16 eigenmodes have been subtracted whereas the exponential form of the correlators does not suffer from this modification. An exception is the pion correlator 
for which the lowest Dirac eigenmodes are crucial, its exponential behavior rapidly gets lost when subtracting low modes. We conclude that the low lying Dirac modes - although providing an important signal for spontaneous chiral symmetry breaking - are not important for the binding mechanism of quarks at least in the isovector mesons (with the exception of the pion).
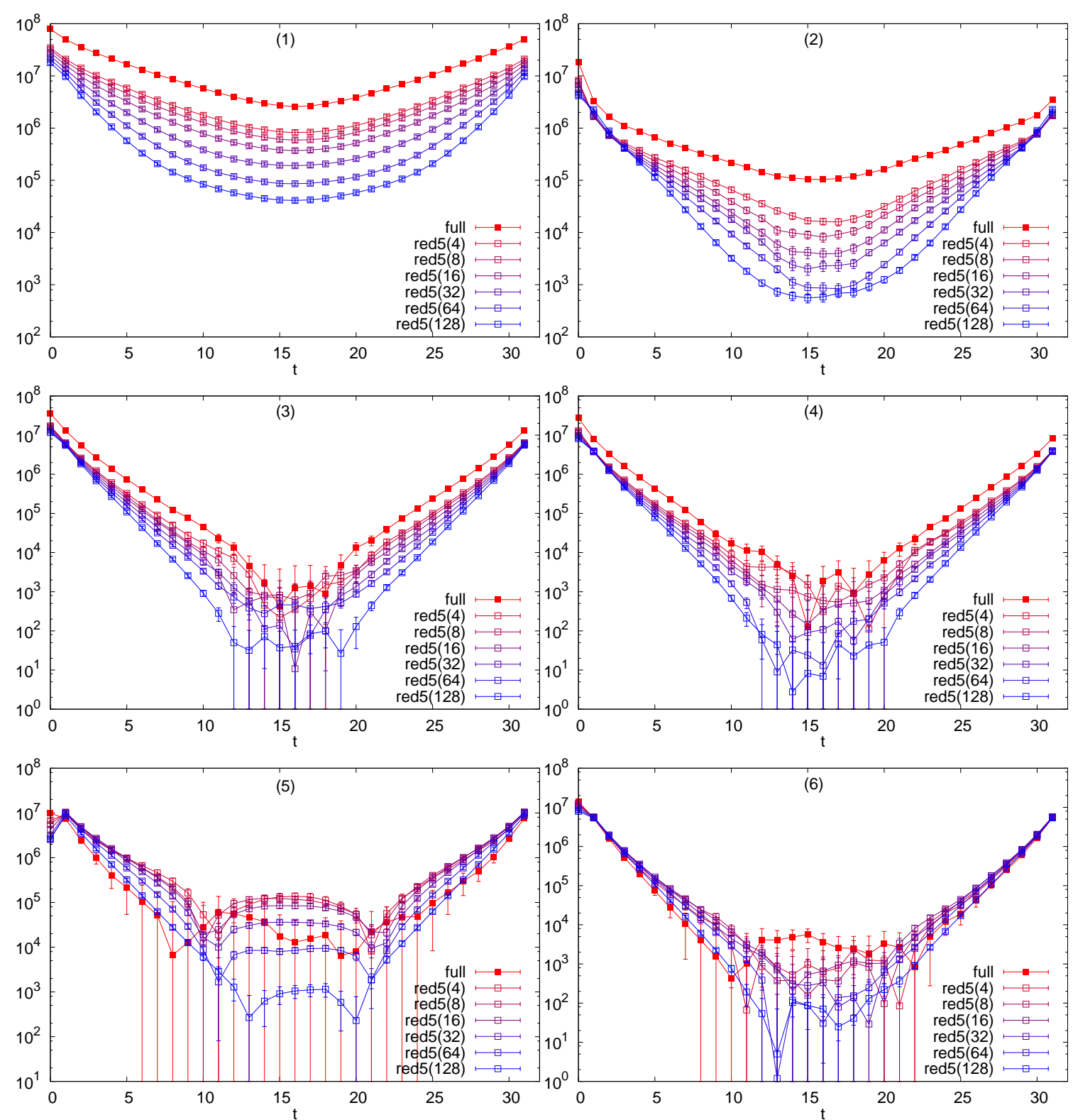

Figure 5: Correlators under low mode reduction. The number in parentheses indicates which current is considered, cf. Table 1.

\section{Acknowledgments}

We would like to thank L. Glozman for helpful discussions. The calculations have been performed on the SGI Altix 4700 of the LRZ Munich and on clusters at ZID at the University of Graz. 
Support by DFG SFB-TR55 and by Austrian Science Fund (FWF) DK W1203-N16 is gratefully acknowledged. M.S. is supported by the Research Executive Agency (REA) of the European Union under Grant Agreement PITN-GA-2009-238353 (ITN STRONGnet).

\section{References}

[1] T. Banks and A. Casher, Chiral symmetry breaking in confining theories, Nucl. Phys. B 169 (1980) 103.

[2] T. A. DeGrand and A. Hasenfratz, Low lying fermion modes, topology and light hadrons in quenched QCD, Phys. Rev. D 64 (2001) 034512, [hep-lat/ 0012021 ].

[3] T. DeGrand, Short distance current correlators: Comparing lattice simulations to the instanton liquid, Phys. Rev. D 64 (2001) 094508, [hep-lat/ 0106001 ].

[4] T. A. DeGrand, Eigenvalue decomposition of meson correlators, Phys. Rev. D 69 (2004) 074024, [hep-ph/0310303].

[5] T. DeGrand and S. Schaefer, Improving meson two-point functions in lattice QCD, Comput. Phys. Commun. 159 (2004) 185-191, [hep-lat/ 0401011 ].

[6] T. DeGrand and S. Schaefer, Improving meson two-point functions by low-mode averaging, Nucl. Phys. (Proc. Suppl.) 140 (2005) 296, [hep-lat/ 0409056 ].

[7] L. Giusti, P. Hernández, M. Laine, P. Weisz, and H. Wittig, Low-energy couplings of QCD from current correlators near the chiral limit, JHEP 04 (2004) 013, [hep-lat/ 0402002 ].

[8] H. Neuberger, Exactly massless quarks on the lattice, Phys. Lett. B 417 (1998) 141, [hep-lat/9707022].

[9] H. Neuberger, More about exactly massless quarks on the lattice, Phys. Lett. B 427 (1998) 353, [hep-lat/9801031].

[10] C. B. Lang and M. Schröck, Unbreaking chiral symmetry, Phys. Rev. D 84 (2011) 087704, [arXiv:1107.5195].

[11] L. Y. Glozman, Restoration of chiral and $U(1)_{A}$ symmetries in excited hadrons, Phys. Rep. 444 (2007) 1, [hep-ph/0701081].

[12] C. Gattringer, C. Hagen, C. B. Lang, M. Limmer, D. Mohler, and A. Schäfer, Hadron spectroscopy with dynamical chirally improved fermions, Phys. Rev. D 79 (2009) 054501, [arXiv: 0812 .1681].

[13] G. P. Engel, C. B. Lang, M. Limmer, D. Mohler, and A. Schäfer, Meson and baryon spectrum for QCD with two light dynamical quarks, Phys. Rev. D 82 (2010) 034505, [arXiv: 1005.1748 ].

[14] C. Gattringer, A new approach to Ginsparg-Wilson fermions, Phys. Rev. D 63 (2001) 114501, [hep-lat/0003005].

[15] C. Gattringer, I. Hip, and C. B. Lang, Approximate Ginsparg-Wilson fermions: A first test, Nucl. Phys. $B 597$ (2001) 451, [hep-lat/ 00070 42].

[16] R. B. Lehoucq, D. C. Sorensen, and C. Yang, ARPACK Users' Guide: Solution of large-scale eigenvalue problems with implicitly restarted Arnoldi methods. SIAM, New York, 1998. 